\title{
Benign Intracranial Hypertension: A Clinical Lesson
}

Major M R Pampapathi
MBBS, FRCS, FRCS(O), RAMC

Senior Specialist in Ophthalmology

Major J B L Kabuubi

MB, BS Acad, DIP Biochem, MRCP, RAMC

British Military Hospital, Munster

SUMMARY: The case of a young woman who suffered from benign intracranial hypertension is described. The clinical history, examination and investigations indicated no cause for her disease, other than obesity. Treatment with corticosteroids prolonged her symptoms and signs, and she only responded to repeated lumbar puncture after dexamethosone was stopped. The lesson we learned was that corticosteroids should rarely be given in benign intracranial hypertension.

\section{Introduction}

A patient with a classical presentation of benign intracranial hypertension (BIH) was initially managed successfully with dexamethasone and a diuretic. Within three weeks her symptoms recurred but did not respond to dexamethasone and periodic lumbar punctures. It was decided to stop dexamethasone and continue with serial lumbar punctures only, which resulted in a dramatic improvement and the avoidance of neurosurgical intervention. The lesson is that serial lumbar punctures, not corticosteroids, are the first line of management of BIH. Even a diuretic on its own may be enough.

\section{Case Report}

A 23 year old Caucasian lady presented with a four weeks' history of headache, worse on changing posture, and a four day history of photophobia, blurred vision, nausea and vomiting. There was no relevant past medical history apart from toxaemia of pregnancy. She was not taking any medication. On examination she was obese (78 kg, height $1.57 \mathrm{~m}$ ), normotensive, (BP 130/90) and had classical bilateral papilloedema (Figure 1), with normal visual acuity, but both blind spots were enlarged. There were no other localising neurological signs. Baseline investigations including a full blood picture, sedimentation rate, urea and electrolytes and skull $\mathrm{X}$ rays were normal. An immediate brain CAT scan showed bilateral cerebral hemispheric oedema with narrow ventricles but no space occupying lesion (Figure 2). A diagnosis of benign intracranial hypertension was made. A lumbar puncture produced normal CSF biochemically and microbiologically, but the CSF pressure was elevated at $37.5 \mathrm{~cm}$. A local civilian neurologist recommended treatment with dexamethasone and acetazolamide. Twenty-four hours afterwards she developed double vision due to a partial right lateral rectus palsy. Several haemorrhages in the right disc were noted. Her headache, however, resolved after seventytwo hours on the above therapy. The diuretic was discontinued because of a rising blood urea. Within four weeks she was completely asymptomatic, and the papilloedema was nearly resolved with only mild blurring of the left disc margin. All cranial nerves were normal as were both blind spots. She returned to the UK because her husband was posted but, unfortunately, within three weeks after stopping. dexamethasone, she had a recurrence of her symptoms: headache and double vision. On admission to the Queen Elizabeth Hospital, $\mathbb{\mathbb { \Phi }}$ Woolwich right lateral rectus palsy and haemorrhage in $\frac{\circ}{\mathbb{0}}$ the right disc, but no papilloedema, were noted. Dexamethasone was re-prescribed, but within a week she developed bilateral lateral rectus palsy and papilloedema with enlarged blind spots. Serial lumbar punctures to remove CSF while on dexamethasone dido not improve her clinical state or lower CSF pressure.? Ventriculo-atrial shunting was considered, but, when dexamethasone was reduced and stopped, periodic lumbar punctures produced rapid clinical improvement and normal CSF pressure. When last reviewed nearly eight months after initial presentation, and having been off corticosteroids for at least three months, she was completely asymptomatic, had no papilloedema, and the visual fields and blind spots were normal, but there was a slight residual right lateral rectus palsy.

\section{Discussion}

Bilateral papilloedema usually is suggestive of serious neurological disease such as a space occupying lesion. It many, however, rarely be due to benign intracranial hypertension in which increased cerebrospinal fluid pressure occurs in the absence of localising neurological signs, intracranial space occupying lesion or cerebrospinal outflow obstruction (internal or external hydrocephalus). BIH may be found in association with lateral cavenous sinus thrombosis secondary to otitis media; hypervitaminosis A; hyperparathyroidism; drugs such as the contraceptive pill, tetracycline, malidixic acid, corticosteroid therapy or its withdrawal and obesity. The association with obesity is not clear ${ }^{1}$. Bilateral papilloedema in carbon dioxide retention and polycythaemia rubra vera is well known. BIH is often 


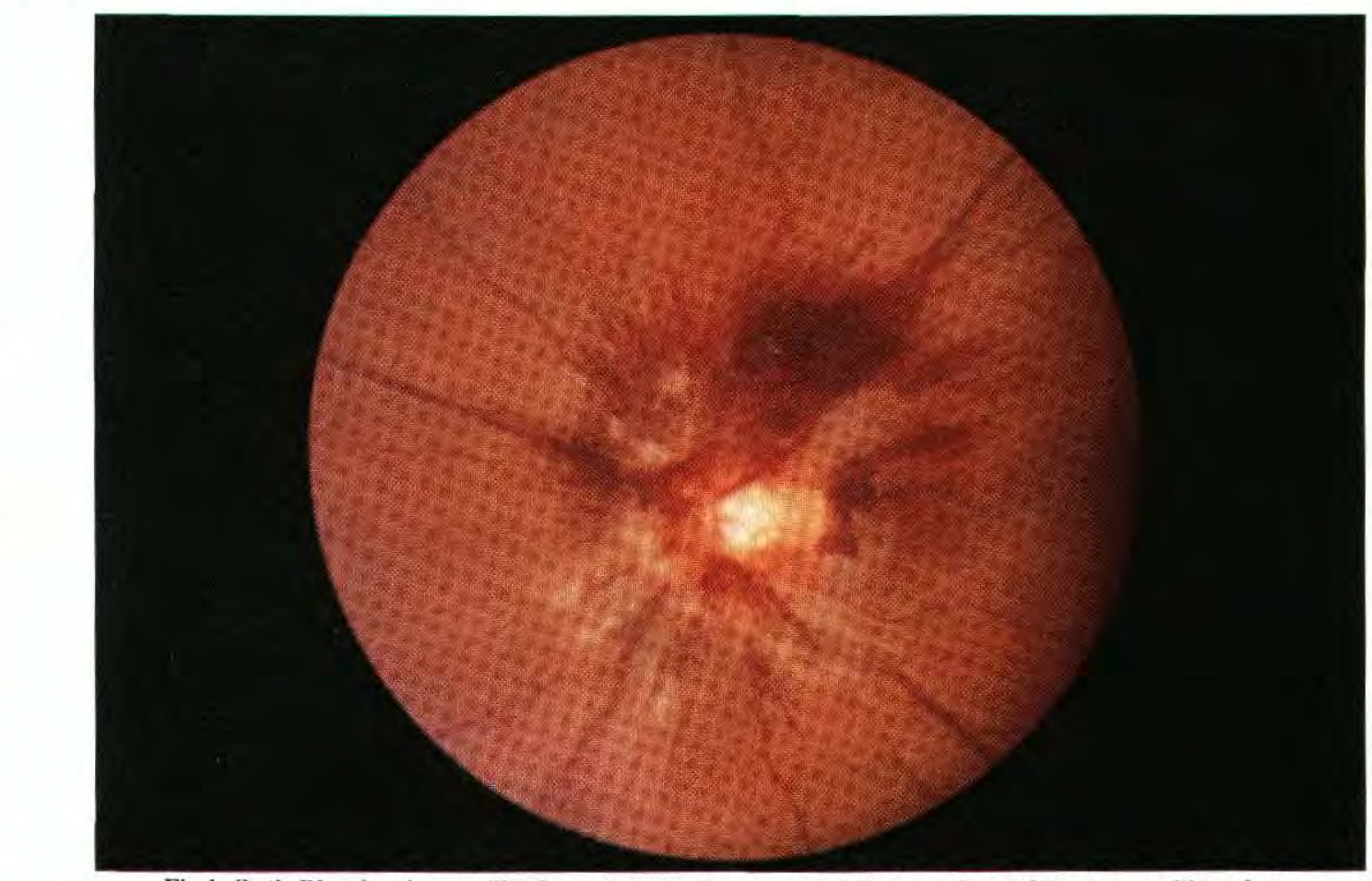

Fig 1. Optic Disc showing papilloedema, haemorrhages around disc margins and "cotton wool" exudates.
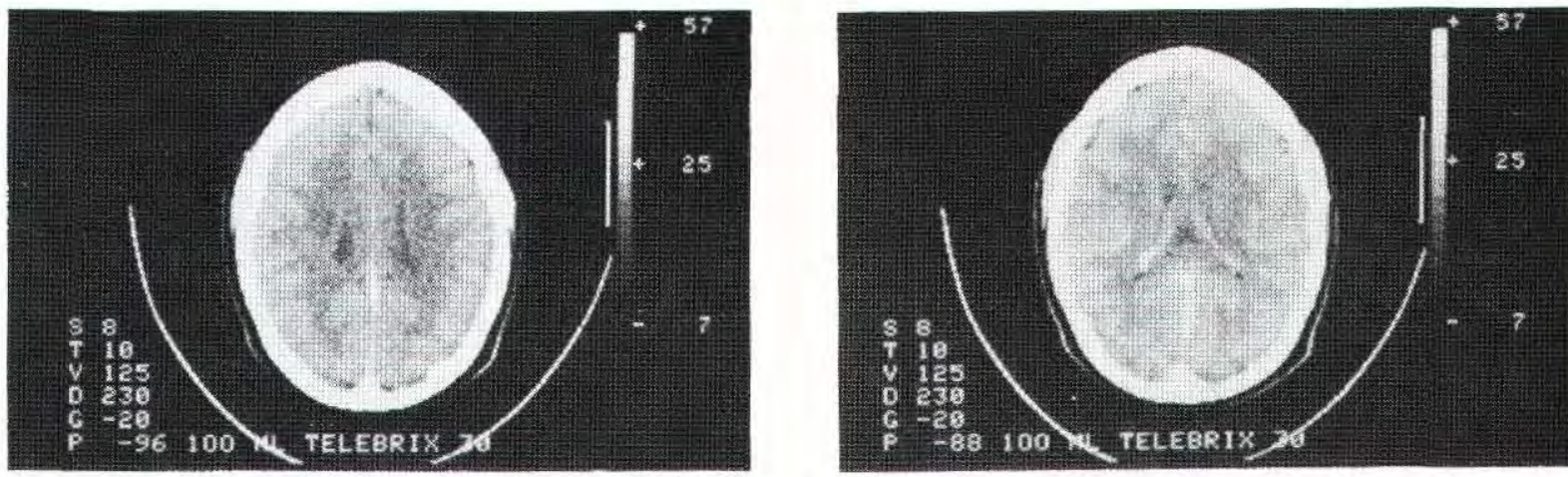

Fig 2. CAT Scans showing brain oedema, narrow ventricles and no evidence of space occupying lesions. 
seen after head injury in children but not in adults. It is most common from childhood to middle age, usually in obesc females. The diagnosis of BIH is made by exclusion. The pathophysiology may be cither due to an increase in dural sinus pressure, decreased absorption of CSF (for which there is some evidence ${ }^{2.3}$ ), interstitial brain oedema, or a combination of all three.

$\mathrm{BIH}$ is generally a self limiting disease. The CSF pressure usually returns to normal within six to twelve weeks with improvement of symptoms but a small group of patients show elevated CSF pressure despite improvement in symptoms.

The feared complication of BIH is visual failure. Therefore treatment is only indicated either to improve symptoms or, in a small number of patients, to prevent visual failure. Although our patient initially appeared to improve when started on dexamethasone she developed double vision and right lateral rectus nerve palsy twentyfour hours after starting corticosteroids, and bilateral lateral rectus norve palsy and papilloedema when dexamethasone was re-prescribed. This suggests that dexamethasone increased brain swelling, causing entrapment of the 6th cranial nerves and a recurrence of papilloedema, thus illustrating the association between corticosteroid therapy/withdrawal and BIH. Corticosteroids are not the first line of management of $\mathrm{BIH}$ in the UK because they are known to cause the disease and once started they are difficult to stop because of the recurrence of symptoms and signs of BIH on withdrawing the drug. They also cause obesity which is a common association with BIH. We had to stop dexamethasone altogether before our patient responded. Corticosteroids can add hazards to a condition which is self limiting, especially when given to a patient with an undiagnosed space occupying lesion; the symptoms and signs may disappear making the diagnosis impossible and prognosis grave.

The first line of management of BIH is a diuretic, preferably a thiazide with potassium supplement, but not acetazolomide, and this may be sufficient to control the disease $e^{4}$. If there is no improvement, serial lumbar punctures aimed at withdrawing a smaller quantity of CSF (up to $10-20 \mathrm{ml}$ ) at a time is the next alternative. The aim is to lower the CSF pressure by half, or to return it to normal. This can be done by daily lumbar puncture during the first week then thrice, twice, and once a week while monitoring the CSF pressure and the blind spot area $^{4}$. This may work by providing a leak in the spinal musculature. Surgery may rarely have a place in managing patients with $\mathrm{BIH}$ who have not responded to conservative measures especially if the vision is threatened.

Before our patient responded to repeated lumbar puncture, ventricular-atrial shunting was contemplated. Narrow ventricles make this operation difficult. Subtemporal decompression or incision of the optic sheath $^{5}$ is rarely indicated and only if the vision is threatened or when other measures have failed, but the morbidity is high and this operation is not often done nowadays ${ }^{h}$.

\section{Acknowledgement}

We wish to thank Mr B H Schurr and Dr Findley for their useful advice and criticism, Brigadier Crawford for allowing us to report on the patient who was under our joint care, Colonel C R Winfield for his help in preparing this manuscript and Mrs Caroline Fisher for her secreta rial help.

\section{REFERENCES}

1. GREER M. Benign Intracranial Hypertension Obesity. Neurology (Minneap) 1965; 15:382-388.

2. Johnston I. The reduced CSF Absoption Syndrome Lancel 1973; 2: 418-420.

3. Jolnston I and PAterson A. Benign Intracranial Hyper tension.

(i) Diagnosis and Prognosis. Brain 1974; 97: 289-300.

(ii) CSF Pressure and Circulation. Brain 1974; 97: 301-312.

4. JefFerson A and Clark J. Treatment of Benign Intracranial Hypertension by Dehydrating Agents with particular reference to the measurement of the blind spot area as a means of recording improvement. J Neurosurg Psychial 1976; 39: 627-639.

5. Gallaraith J E K and Sullivan J H. Decompression of perioptic meninges for relief of papillocdema. Am J Ophth 1973; 76: 687-692.

6. Jefrerson A. Oxford Textbook of Medicine by Weatherall, Leadington and Warrell 1984; 2:21-81. 